\title{
Extremely massive disc galaxies in the nearby Universe form through gas-rich minor mergers
}

\author{
R. A. Jackson, ${ }^{1 \star}$ S. Kaviraj, ${ }^{2}$ G. Martin, ${ }^{3,4}$ J. E. G. Devriendt, ${ }^{5}$ E. A. Noakes-Kettel, ${ }^{2}$ \\ J. Silk, ${ }^{5,6,7}$ P. Ogle ${ }^{8}$ and Y. Dubois ${ }^{6}$ \\ ${ }^{1}$ Department of Astronomy and Yonsei University Observatory, Yonsei University, Seoul 03722, Republic of Korea \\ ${ }^{2}$ Centre for Astrophysics Research, School of Physics, Astronomy and Mathematics, University of Hertfordshire, Hatfield, AL10 9AB, UK \\ ${ }^{3}$ Korea Astronomy and Space Science Institute, 776 Daedeokdae-ro, Yuseong-gu, Daejeon 34055, Korea \\ ${ }^{4}$ Steward Observatory, University of Arizona, 933 N. Cherry Ave, Tucson, AZ 85719, USA \\ ${ }^{5}$ Dept of Physics, University of Oxford, Keble Road, Oxford OX1 3RH UK \\ ${ }^{6}$ Institut d'Astrophysique de Paris, Sorbonne Universités, UMPC Univ Paris 06 et CNRS, UMP 7095, 98 bis bd Arago, 75014 Paris, France \\ ${ }^{7}$ Department of Physics \& Astronomy, The Johns Hopkins University, Baltimore, MD 21218, USA \\ ${ }^{8}$ Space Telescope Science Institute, 3700 San Martin Drive, Baltimore, MD 21218, USA
}

25 January 2022

\begin{abstract}
In our hierarchical structure-formation paradigm, the observed morphological evolution of massive galaxies - from rotationally-supported discs to dispersion-dominated spheroids - is largely explained via galaxy merging. However, since mergers are likely to destroy discs, and the most massive galaxies have the richest merger histories, it is surprising that any discs exist at all at the highest stellar masses. Recent theoretical work by our group has used a cosmological, hydrodynamical simulation to suggest that extremely massive $\left(\mathbf{M}_{*}>10^{11.4}\right.$ $\mathrm{M}_{\odot}$ ) discs form primarily via minor mergers between spheroids and gas-rich satellites, which create new rotational stellar components and leave discs as remnants. Here, we use UV-optical and HI data of massive galaxies, from the SDSS, GALEX, DECaLS and ALFALFA surveys, to test these theoretical predictions. Observed massive discs account for $\sim 13$ per cent of massive galaxies, in good agreement with theory ( $\sim 11$ per cent). $\sim 64$ per cent of the observed massive discs exhibit tidal features, which are likely to indicate recent minor mergers, in the deep DECaLS images (compared to $\sim 60$ per cent in their simulated counterparts). The incidence of these features is at least four times higher than in low-mass discs, suggesting that, as predicted, minor mergers play a significant (and outsized) role in the formation of these systems. The empirical star-formation rates agree well with theoretical predictions and, for a small galaxy sample with HI detections, the HI masses and fractions are consistent with the range predicted by the simulation. The good agreement between theory and observations indicates that extremely massive discs are indeed remnants of recent minor mergers between spheroids and gas-rich satellites.
\end{abstract}

Key words: galaxies: spiral - galaxies: evolution - galaxies: formation - galaxies: interactions

\section{INTRODUCTION}

Observational studies of the morphology of massive galaxies show that, while discs dominate the high-redshift Universe, the morphologies of nearby massive galaxies are mostly spheroidal in nature (Bernardi et al. 2003; Wuyts et al. 2011; Ryan et al. 2012; Conselice et al. 2014; Buitrago et al. 2014; Shibuya et al. 2015). In our standard hierarchical structure-formation paradigm, this morphological change, from rotationally-supported discs to dispersion-dominated spheroids, is largely explained by galaxy merging (Toomre 1977;

^ E-mail: r.jackson@yonsei.ac.kr
Barnes 1992; Bournaud et al. 2007; Di Matteo et al. 2007; Oser et al. 2010; Kaviraj 2010; Kaviraj et al. 2011; Dubois et al. 2013, 2016; Lofthouse et al. 2017; Welker et al. 2018; Martin et al. 2018). The large gravitational torques produced during merger events are capable of randomising the ordered rotational orbits of stars within the merging progenitors and creating dispersion-dominated systems (e.g. Springel \& Hernquist 2005; Hilz et al. 2013; Font et al. 2017; Martin et al. 2018, 2019).

The role of merging is thought to become increasingly more important at higher stellar masses. In particular, significant merging activity is considered essential for galaxies to achieve the highest stellar masses (e.g. Faber et al. 2007; McIntosh et al. 2008; Cattaneo 
et al. 2011) beyond the knee of the galaxy mass function $\left(\mathbf{M}_{*} \gtrsim\right.$ $10^{10.8} \mathrm{M}_{\odot}$; Li \& White 2009; Kaviraj et al. 2017), because star formation via direct gas accretion is no longer sufficient to drive the requisite stellar mass growth. However, since mergers can destroy discs, and the most massive galaxies have the richest merger histories, it is surprising that both observational (e.g. Conselice 2006; Ogle et al. 2016, 2019) and theoretical (e.g. Martin et al. 2018; Jackson et al. 2020) studies suggest that a significant minority of galaxies at the highest stellar masses $\left(\mathrm{M}_{*}>10^{11.4} \mathrm{M}_{\odot}\right)$ have discy morphologies.

If discs exist in the stellar mass regime when mergers are frequent, their merger histories must involve peculiarities that either rejuvenate discy components or allow these discs to survive the mergers themselves. For example, theoretical work has shown that in gas-rich mergers, the gas brought in by the progenitors can create new discy stellar components in the remnants (e.g. Springel \& Hernquist 2005; Robertson et al. 2006; Governato et al. 2009; Hopkins et al. 2009; Font et al. 2017; Martin et al. 2018; Peschken et al. 2019). In Jackson et al. (2020, J20 hereafter) we have used Horizon-AGN, a cosmological hydrodynamical simulation (Dubois et al. 2014; Kaviraj et al. 2017), to probe the potential channels by which massive discs may form in the standard paradigm. J20 showed that extremely massive $\left(\mathrm{M}_{*}>10^{11.4} \mathrm{M}_{\odot}\right)$ discs do exist in the simulation and are created via two channels. In the primary channel, which accounts for $\sim 70$ per cent of these systems, a significant merger with a mass ratio greater than 1:10, between a massive spheroid and a gas-rich satellite, 'spins up' the spheroid by creating a new rotational stellar component, and leaves a massive disc as the remnant. In the secondary channel, a system maintains a disc throughout its lifetime, due to an anomalously quiet merger history, with a merger rate that is more than a factor of two lower than in galaxies of a comparable stellar mass. This enables the galaxy to retain its gas reservoir more easily.

Several studies in the literature have also explored the formation mechanisms of massive disc galaxies. Martig et al. (2021) have looked at the formation history of NGC 5746 (an edge-on disc galaxy with $\mathrm{M}_{\star} \sim 10^{11} \mathrm{M}_{\odot}$ ). They find that this galaxy formed its massive disc early and only underwent one significant merger ( $\sim 1: 10$ mass ratio), without disruption to the disc component of the galaxy (consistent with one of the channels for massive disc formation outlined in J20). Ogle et al. (2016, 2019) have suggested that the local massive discs in their study may have formed via major mergers (mass ratios greater than 1:4) between two gas-rich spiral galaxies. Monachesi et al. (2016) studied the outskirts of massive disc galaxies, finding variations in the median colours as a function of radius. They conclude that this is indicative of several small, accreted objects which have built up the outskirts of these galaxies (consistent with the minor-merger hypothesis in J20). Zeng et al. (2021) have used the Illustris-TNG simulation (Nelson et al. 2019), to show that disc galaxies in the mass range $\left(\mathrm{M}_{\star}>8 \times 10^{10} \mathrm{M}_{\odot}\right)$ form via three channels. They find that $\sim 8$ per cent of such discs have a quiescent merger history and remain discs throughout their lifetime, whilst $\sim 54$ per cent have a significant increase in their bulge components before later becoming discs again. In their third channel, such discs experience prominent mergers but survive to remain disc-like. However, it is worth noting that this study includes many galaxies with significantly lower stellar mass than those in J20, which likely influences the creation channels and makes it difficult to directly compare the two studies.

If massive discs are indeed formed via the channels suggested in $\mathrm{J} 20$, the simulation makes some specific predictions that are testable. For example, J20 predicts that massive discs should com- prise $\sim 11$ per cent of the massive galaxy population. Since they are typically created via recent minor mergers, a large fraction of these galaxies should exhibit tidal features in deep optical images. Given the gas-rich nature of these mergers, J20 suggests that these systems should show reasonably high star-formation rates (SFRs) of a few solar masses per year. Finally, since massive ellipticals are predicted to have their last significant mergers at higher redshifts, and since these mergers are not gas-rich, both the fraction of tidally-disturbed galaxies and their SFRs should be elevated in the massive discs, compared to that in their spheroidal counterparts.

The purpose of this observational paper is to confront the theoretical predictions from $\mathrm{J} 20$ with multi-wavelength survey data, in order to establish whether the predictions are indeed supported by the observations. This paper is structured as follows. In Section 2, we describe the construction of a sample of massive galaxies and their multi-wavelength data, using the Sloan Digital Sky Survey (SDSS; York et al. 2000; Abazajian et al. 2009), the Dark Energy Camera Legacy Survey (DECaLS; Dey et al. 2019), the Galaxy Evolution Explorer (GALEX; Morrissey et al. 2007) and the Arecibo Legacy Fast ALFA (ALFALFA; Haynes et al. 2011) survey. We also describe the process of classifying galaxy morphology and identifying objects which have tidal features via visual inspection. In Section 3, we compare the observed properties of the massive discs in our sample with the predictions of J20. We summarise our findings in Section 4.

\section{A SAMPLE OF MASSIVE GALAXIES IN THE NEARBY UNIVERSE}

We construct our sample of nearby massive galaxies from the SDSS using the MPA-JHU value-added catalog ${ }^{1}$. We select objects which have SDSS spectroscopic redshifts in the range $0.03<z<0.1$ and where the lower limit of their stellar masses, taken from the MPAJHU catalog (Brinchmann et al. 2004), is greater than $10^{11.4} \mathrm{M}_{\odot}$. We identify 708 galaxies which match these criteria.

The lower limit of the mass range ensures that our empirical sample of massive galaxies is comparable to those in the theoretical study of J20. The upper limit of the redshift range produces a sample with a median redshift that matches that of the simulated galaxies in J20. Standard depth SDSS imaging is typically too shallow to reveal faint tidal features from minor mergers (e.g. Kaviraj 2010, 2014b). Instead, we use images from DECaLS, which provides deep optical imaging over $\sim 14,000$ square degrees in the northern hemisphere. The DECaLS images in the $g, r$ and $z$ bands have $5 \sigma$ point-source depths of $\sim 24.0, \sim 23.4$ and $\sim 22.5$ magnitudes respectively, roughly 1.5 magnitudes deeper than their SDSS counterparts, with similar seeing. At our redshifts of interest, the impact of surface-brightness dimming is still relatively minor, making it possible to see faint structures like tidal features in deep optical images (e.g. Kaviraj et al. 2019).

To demonstrate the improvement in our ability to detect mergerinduced tidal features in deeper imaging, we show, in Figure 1, images of the same galaxy from the SDSS, DECaLS and the Hyper Suprime-Cam Subaru Strategic Program (HSC-SSP Aihara et al. 2019), which is a further 2 mags deeper than DECaLS. While the tidal features are invisible in the SDSS image, they are progressively more visible in the DECaLS and HSC-SSP images (and clearest in

\footnotetext{
1 https://wwwmpa.mpa-garching.mpg.de/SDSS/DR7/
} 
the deepest HSC-SSP image). Note that, while the HSC-SSP is the deepest wide-area optical survey currently available, its footprint $\left(\sim 1500 \mathrm{deg}^{2}\right)$ is considerably smaller than that of DECaLS and therefore not suitable for this study. Indeed, only 4 out of the 708 massive galaxies in our sample are in the HSC-SSP footprint.

We use SFRs from the GALEX-SDSS-WISE Legacy Catalog (GSWLC; Salim et al. 2016), which are derived via spectral energy distribution (SED) fitting using total magnitudes from the GALEX and SDSS surveys. SFRs derived using total magnitudes are more likely to be representative of the total SFR of the system than those derived, for example, using emission lines measured within the SDSS fibre (which will only sample the star formation activity in the central regions of the galaxy). These total SFRs are more appropriate for comparison to the SFRs measured in simulated galaxies, where the entire galaxy is used to measure the SFR. Finally, we use HI masses from the ALFALFA survey.

\subsection{Morphological classification and identification of merger-induced tidal features}

We visually inspect the composite $g r z$ images of each individual massive galaxy from DECaLS to morphologically classify it as either a spheroid (which includes ellipticals and lenticular systems) or a disc. For each system, we also flag the presence of tidal features. In Figure 2, we show a representative sample of galaxies, classified as spheroids (left-hand panel) and discs (right-hand panel) respectively. The top row of each panel shows examples of galaxies classified as 'disturbed' (i.e. those which exhibit the presence of tidal features), while the bottom row shows galaxies classified as 'relaxed' (i.e. those in which no tidal features are present).

\section{OBSERVED PROPERTIES OF MASSIVE GALAXIES IN THE LOCAL UNIVERSE}

\subsection{Morphological properties and host halo mass}

We begin our analysis by considering the morphological properties of galaxies in our observational sample (summarised in Table 1). $\sim 13$ per cent of our massive galaxies are discs, of which $\sim 64$ per cent show evidence for tidal features (the corresponding value for earlytypes is $\sim 31$ per cent). This is a lower limit because, as indicated by Figure 1, it is likely that the fraction of galaxies with tidal features could be higher in deeper imaging. The corresponding fraction of systems in the low-mass disc population, which show tidal features in SDSS Stripe 82 images (which have similar depth and seeing to DECaLS), is between $\sim 11$ and 17 per cent, depending on the specific morphology of the low-mass discs in question (e.g. $\mathrm{Sa} / \mathrm{Sb} / \mathrm{Sc} / \mathrm{Sd}$, see Kaviraj 2014b). The median stellar mass of the low-mass disc population in Kaviraj (2014b) is $\sim 10^{10.3} \mathrm{M}_{\odot}$ (Kaviraj 2014a). The fraction of tidal features in massive discs is therefore significantly elevated (by at least around a factor of 4) compared to their low-mass counterparts, implying that the formation of these galaxies involves a much higher incidence of recent mergers.

To confirm that the tidal-feature fraction in the observed galaxies is indeed indicative of the formation methods predicted by $\mathbf{J} 20$, we also construct realistic mock images of the massive discs from J20 and estimate the corresponding tidal-feature fraction from the simulation (Figure 3 ). We produce produce two sets of mock $z$-band images using the DECaLS pixel scale ( 0.262 arcseconds) and point

\begin{tabular}{ccc}
\hline \hline 1 & 2 & 3 \\
\hline Morphology & Number fraction & Fraction with tidal features \\
\hline \hline Disc & 0.13 & 0.64 \\
Spheroid & 0.87 & 0.31
\end{tabular}

Table 1. The morphological properties of nearby massive $\left(\mathrm{M}_{*}>10^{11.4} \mathrm{M}_{\odot}\right)$ galaxies. Columns: (1) morphological class (2) number fraction of galaxies in this morphological class (3) fraction of galaxies in this morphological class which exhibits tidal features in the DECaLS images. Massive discs show a high incidence of tidal features (which are significantly elevated, by at least around a factor of 4, compared to their low-mass counterparts), suggesting that the formation of these systems involves a high prevalence of mergers, consistent with the theoretical predictions in J20.

spread function (1.10 arcseconds). The first imposes the DECaLS surface brightness limit ( $27.9 \mathrm{mag} \operatorname{arcsec}^{-2}$, Hood et al. 2018) while the second has no limit. Visual inspection of these images shows that the surface-brightness-limited images exhibit a tidal feature fraction of $\sim 60$ per cent, which is comparable to the fraction ( $\sim 64$ per cent) in the observed images. However, if the images without a surface-brightness limit are used the tidal feature fraction increases to $\sim 74$ per cent, demonstrating that faint tidal features can be missed even at the depth of DECaLS (as illustrated in Figure 1) ${ }^{2}$.

The high frequency of tidal features in massive discs suggests that most of these interactions are unlikely to be major mergers (mass ratios greater than 1:4). This is because, in our mass range of interest, the major merger fraction at low redshift is only a few per cent (e.g. Darg et al. 2010; Mundy et al. 2017), with the tidal features from such interactions remaining visible for 2-3 Gyrs at the depth of the DECaLS images (e.g. Mancillas et al. 2019). The majority of these events are therefore likely to be minor mergers (mass ratios between $1: 4$ and $1: 10)^{3}$, which are typically $4-5$ times more frequent than their major counterparts (e.g. Jogee et al. 2009; López-Sanjuan

\footnotetext{
2 A recent study by Blumenthal et al. (2020), using mock SDSS images, has suggested that tidal features may not be readily visible from either major or minor mergers. It is important to note, however, that Blumenthal et al. consider galaxy pairs with a total stellar mass of $10^{9} \mathrm{M}_{\odot}$ or greater. In our study, the galaxy pair has a mass of at least $10^{11.4} \mathrm{M}_{\odot}$. Indeed, the satellites that are accreted by the larger progenitors in the events that create the massive discs are themselves likely to have stellar masses around $10^{10.8}$ $\mathrm{M}_{\odot}$ (see the analysis in Section 3.2). Tidal features are brighter when the objects involved are more massive, simply because there is more material in the features (e.g. Kaviraj 2014b). Hence the Blumenthal et al. study, which will be dominated by mergers between much lower mass galaxies, as a result of the shape of the galaxy stellar mass function (e.g. Wright et al. 2017), is not comparable to our work. As we note in our analysis above, tidal features are very common in galaxies in the mass range of interest in this study, in the Horizon-AGN simulation.

${ }^{3}$ It is worth noting that the tidal features seen around the massive discs are unlikely to be the result of fly-bys. All massive discs in the J20 study are the result of actual galaxy mergers and not fly-bys, which cannot produce the strong morphological transformation required for the creation of these systems. In terms of the presence of tidal features, while low-mass $\left(\mathrm{M}_{\star}<10^{8}\right.$ $\mathrm{M}_{\odot}$ ) dwarf galaxies can sometimes produce tidal features as a result of very close fly-bys (e.g. Jackson et al. 2021), these features already become rare in fly-by events involving relatively massive satellites with stellar masses around $10^{9} \mathrm{M}_{\odot}$ (Martin et al. 2021). More massive satellites (such as the ones involved in the creation of the massive discs, see text in Section 3.2) are therefore extremely unlikely to produce tidal features.
} 


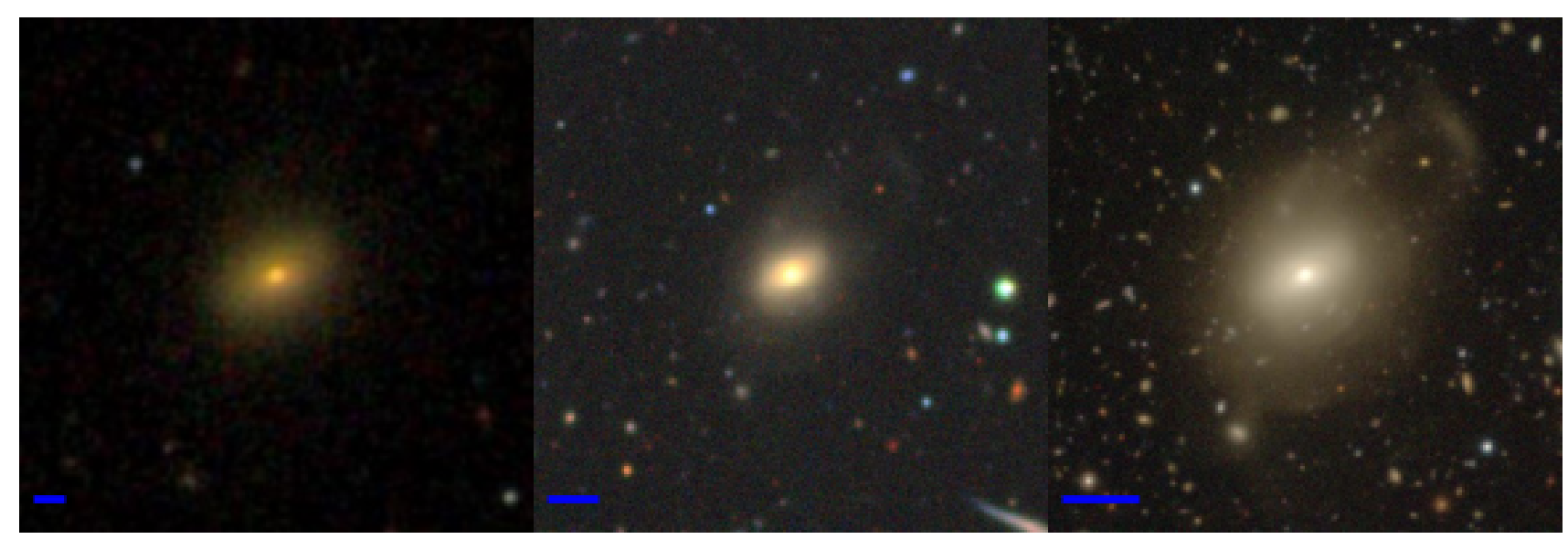
SDSS DECaLS

Figure 1. Images of the same galaxy from the SDSS, DECaLS and the HSC-SSP Wide surveys. DECaLS and HSC-SSP are $\sim 1.5$ and $\sim 4$ mags deeper than the SDSS respectively. While the tidal features are invisible in the SDSS, they become progressively more easily visible in the deeper images. This figure highlights the need to use a deep-wide survey like DECaLS to detect galaxies that have recently undergone a merger. The blue bar in the bottom left indicates 10 arcseconds in each image. This figure may look better on screen than in print.
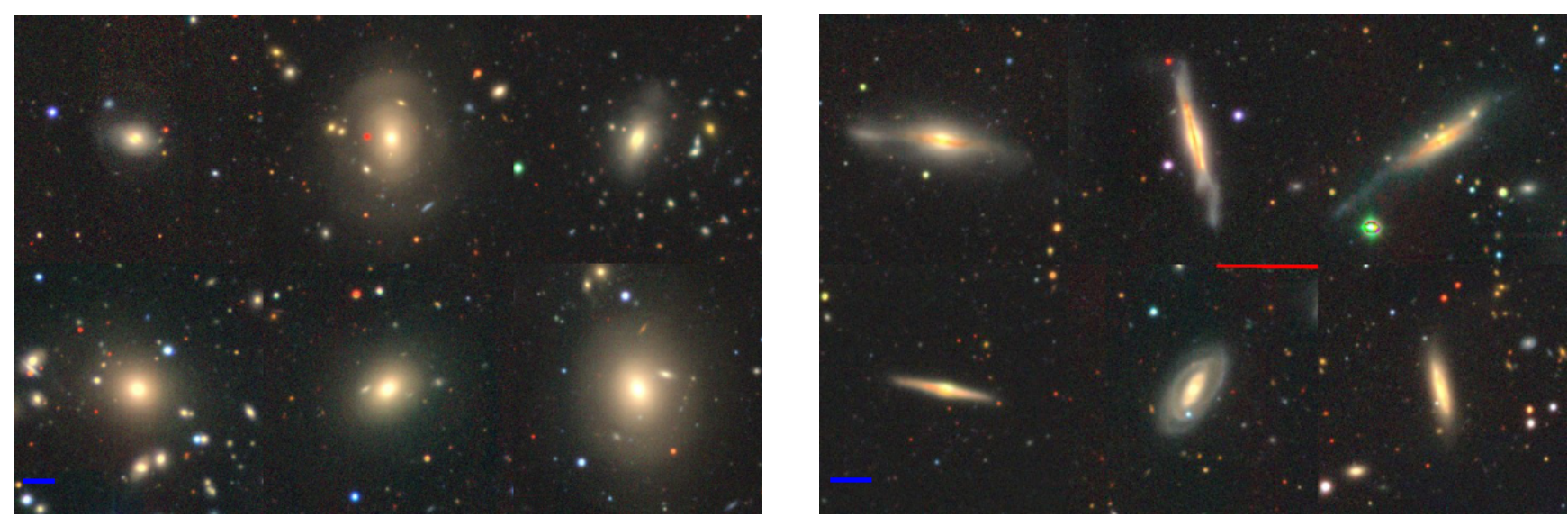

Figure 2. Examples of spheroids (left) and discs (right) from our massive galaxy sample. The images are $g r z$ composites from DECaLS. 'Disturbed' galaxies (i.e. those that show evidence of tidal features such as tidal tails, plumes and prominent shells) are shown in the top row, while 'relaxed' galaxies (i.e. those that do not show evidence of tidal features) are shown in the bottom row. The blue bar in the bottom left panel indicates 10 arcseconds in each image. This figure may look better on screen than in print.

et al. 2010; Lotz et al. 2011; Kaviraj 2014b; Rodriguez-Gomez et al. 2015).

These observational results appear consistent with the theoretical predictions of $\mathbf{J} 20$, which suggest that $\sim 11$ percent of the massive galaxies in the simulation are discs, and that these systems form via recent minor mergers that take place within the last $\sim 2-3$ Gyrs. In addition, massive spheroids in the simulation typically underwent their most recent mergers at higher redshift than their discy counterparts. Given that the tidal features from these mergers will have had more time to fade, this will result in fainter tidal features at $z \sim 0$, which appears consistent with the lower tidal fraction observed in the massive early-types in our observed sample.

Since relatively dense neighbourhoods like groups are likely to favour mergers (e.g. Kaviraj et al. 2015), we consider the local environments of our massive discs, both in the observed sample and their theoretical counterparts. J20 suggest that extremely massive discs usually inhabit relatively massive dark-matter halos (which are indicative of relatively dense environments, such as large groups or clusters). Therefore, if the observed sample is representative of the simulated population, they should also reside in such massive halos. To study the local environment of our observed massive discs, we use the galaxy group catalogue from Yang et al. (2007) which provides halo masses for our observed galaxies.

Figure 4 shows stellar vs halo mass for our observed discs and compares them to their theoretical counterparts from J20. The paucity of simulated systems at very high stellar mass is driven by the fact that the simulation volume is only $\sim 100 \mathrm{Mpc}$ and therefore very rare objects, like galaxies at the highest stellar masses, are absent from the simulation box. Both the observed and simulated galaxies inhabit large halos, with the median stellar and halo mass 


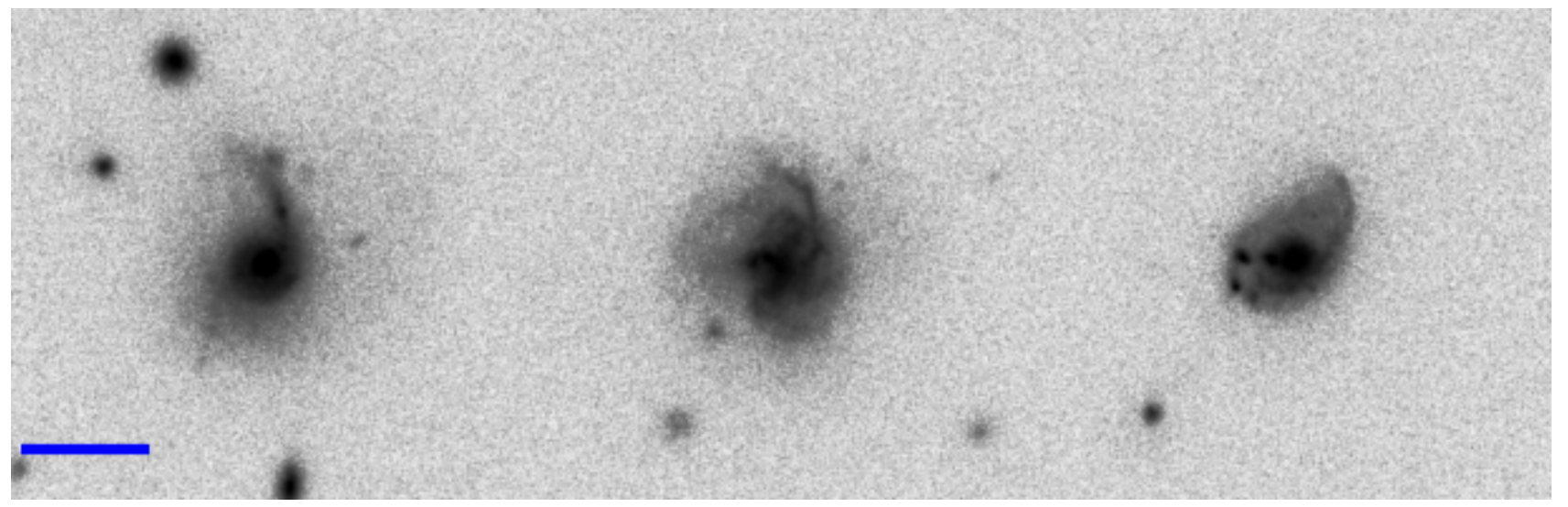

Figure 3. Example $z$-band images of simulated massive disc galaxies (from J20) in the Horizon-AGN simulation. These images are made to match the DECaLS observations, including the surface brightness limit, pixel scale and point spread function. The simulated galaxies show tidal features, as is the case in their observed counterparts. The blue bar in the left panel indicates $25 \mathrm{kpc}$ in each image.

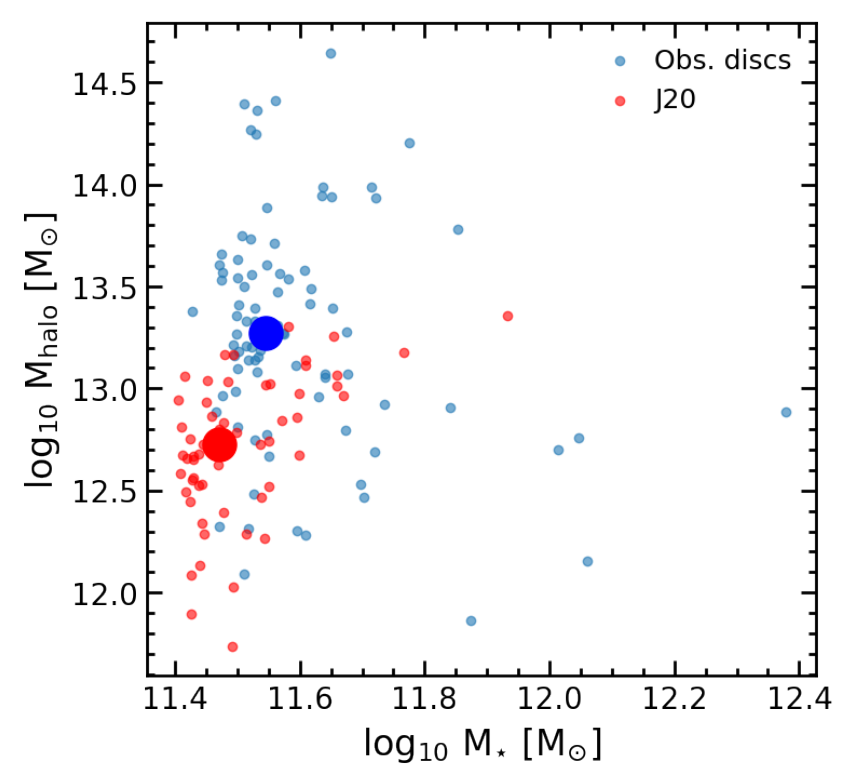

Figure 4. Stellar mass vs dark matter halo mass for our observed massive discs and their theoretical counterparts in J20. The larger points show the median stellar and halo masses for both samples. The halo masses for the observed sample are taken from Yang et al. (2007). The lack of simulated systems at very high stellar mass is due to the fact that the simulation volume is only $\sim 100 \mathrm{Mpc}$ and therefore very rare objects, like the most massive galaxies, are absent from the simulation box. Despite the bias towards larger stellar masses in the observed sample, both populations of massive discs primarily reside inside massive halos, indicating relatively dense environments, which is consistent with the high incidence of tidal features in this population.

of both samples, denoted by the larger points, lying on a locus of increasing stellar and halo mass. To further this argument we compare how the halo masses of the massive discs compare to the distribution of halo masses of all galaxies with $\mathrm{M}_{\star}>10^{10} \mathrm{M}_{\odot}$. We find that both the observed and simulated sample of massive discs occupy above the upper 10 percentile of halo masses. They therefore reside in very massive halos, consistent with the high frequency of tidal features seen in these systems.

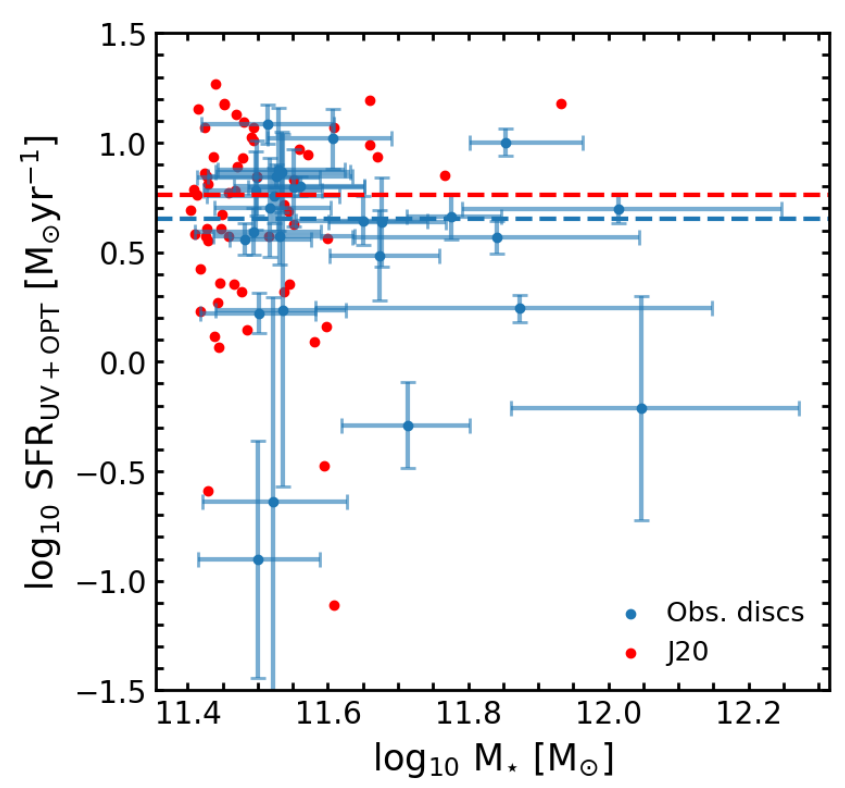

Figure 5. SFR as a function of stellar mass for massive discs in our observational sample (blue) and the simulated sample of J20 (red). Dashed lines indicate median values. The SFRs from the observations and simulations show comparable median values and distributions. While we omit spheroids for clarity, the medians and distributions for all populations are summarised in Table 2. The paucity of simulated systems at very high stellar mass is driven by the fact that the simulation volume is only $\sim 100 \mathrm{Mpc}$ and therefore very rare objects, like galaxies at the highest stellar masses, are absent from the simulation box.

\subsection{Star formation rates and atomic gas properties}

We proceed by considering the SFRs of our observed massive galaxies. If the predicted formation mechanisms of massive discs in J20 are broadly accurate, then the expectation is that the SFRs in the observed and simulated massive discs should be comparable and that the SFRs in the discs should be significantly enhanced compared to that in the spheroids.

In Figure 5, we compare the total SFRs of the observed massive 


\begin{tabular}{|c|c|c|c|c|}
\hline 1 & 2 & 3 & 4 & 5 \\
\hline Morphology & $\begin{array}{c}\log _{10} \mathrm{Sim} . \mathrm{SFR}\left[\mathrm{M}_{\odot} \mathrm{yr}^{-1}\right] \\
{\left[16^{\text {th }} \bullet \text { median } \bullet 84^{\text {th }}\right]}\end{array}$ & $\begin{array}{c}\log _{10} \text { Obs. SFR }\left[\mathrm{M}_{\odot} \mathrm{yr}^{-1}\right] \\
{\left[16^{\text {th }} \bullet \text { median } \bullet 84^{\text {th }}\right]}\end{array}$ & $\begin{array}{l}\log _{10} \text { Sim. sSFR }\left[\mathrm{yr}^{-1}\right] \\
{\left[16^{\text {th }} \bullet \text { median } \bullet 84^{\text {th }}\right]}\end{array}$ & $\begin{array}{l}\log _{10} \text { Obs. sSFR }\left[\mathrm{yr}^{-1}\right] \\
{\left[16^{\text {th }} \bullet \text { median } \bullet 84^{\text {th }}\right]}\end{array}$ \\
\hline Discs & $0.28 \bullet \mathbf{0 . 7 6} \bullet 1.07$ & $0.23 \bullet \mathbf{0 . 6 5} \bullet 0.86$ & $-11.13 \bullet-\mathbf{- 1 0 . 6 8} \bullet-10.36$ & $-11.53 \bullet-\mathbf{1 0 . 9 4} \bullet-10.67$ \\
\hline Spheroids & $-0.73 \bullet \mathbf{0 . 1 2} \bullet 0.68$ & $-0.94 \bullet-\mathbf{0 . 3 7} \bullet 0.31$ & $-12.18 \bullet-\mathbf{- 1 1 . 0 0} \bullet-10.39$ & $-12.56 \bullet \mathbf{- 1 1 . 9 6} \bullet-11.24$ \\
\hline
\end{tabular}

Table 2. SFRs and sSFRs for massive $\left(M_{*}>10^{11.4} M_{\odot}\right)$ galaxies of different morphologies (indicated in column 1). In each column we describe the 16th, 50th (i.e. the median) and 84th percentile values from the SFR and sSFR distributions. Columns are as follows: (2) total SFRs of simulated massive galaxies from J20 (3) total SFRs of the observed massive galaxies, calculated using SED-fitting of UV + optical photometry, from the GSWLC (4) total sSFRs of simulated massive galaxies from J20 (5) total sSFRs of the observed massive galaxies. The median values of the simulated and observed SFRs and sSFRs are in reasonably good agreement with each other for the massive disc population.

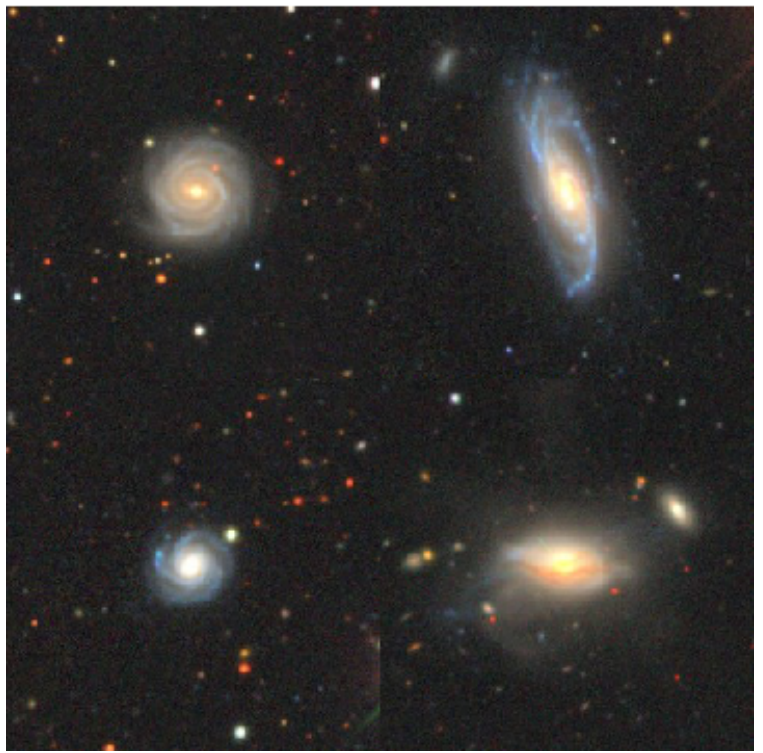

Figure 6. DECaLS images of the 4 galaxies which have HI detections from the ALFALFA survey. All 4 systems are discs, with only 1 showing evidence of tidal features (lower right-hand panel). This disturbed disc has a high signal-to-noise detection in ALFALFA, while the other three have low $\mathrm{S} / \mathrm{N}$ detections, due to their lower HI content (see Figure 7 below).

discs calculated using integrated UV and optical photometry (blue) with the corresponding theoretical predictions (where the total SFR is calculated as an average over $\sim 100 \mathrm{Myrs}$ ) from J20 (red). The choice of 100 Myr is driven by the fact that GALEX UV photometry (which is used to derive the observed SFRs) is most sensitive over this timescale (e.g. Morrissey et al. 2007), making the theoretical and observed SFRs comparable. Both the median values of the observed and theoretical samples (shown using the dashed lines) and the distributions of their SFRs are in good agreement with each other (the spheroids are omitted for clarity). In Table 2, we summarise the theoretical and observed SFRs of our massive galaxies. For completeness, we also show the specific SFRs (sSFRs) of these systems. Finally, we note that, in a similar vein to what is seen in the predictions of J20, the SFRs and sSFRs of the observed massive discs are significantly elevated compared to that in their spheroidal counterparts.

While the agreement between the observed and theoretical SFRs indicates that the gas masses involved in the star formation events are broadly predicted correctly, we explore empirical constraints on what these gas masses are likely to be. While large area surveys of molecular gas are not available, we explore the HI masses of our massive galaxies using the ALFALFA survey. None of the massive spheroids are detected in ALFALFA. However, 4 massive discs (out of 92) have ALFALFA detections. In all cases, both the spatial and velocity offsets between the SDSS and the ALFALFA sources are small, indicating reliable matches. The spatial offsets are all less than 15 arcseconds (the average positional accuracy of ALFALFA is $\sim 24$ arcseconds), while the velocity offsets (calculated from the spectroscopic redshifts and HI velocities) are within $80 \mathrm{~km}$ $\mathrm{s}^{-1}$.

Figure 6 shows DECaLS images of these discs, three of which are relaxed and one is disturbed (lower-right hand panel). Figure 7 indicates that the HI mass (and corresponding $\mathrm{HI}$ fraction, defined as the $\mathrm{HI}$ mass divided by the sum of the $\mathrm{HI}$ and stellar masses) in the disturbed disc is higher than those in its relaxed counterparts. Note that the average mass of the observed massive discs is $10^{11.56}$ $\mathrm{M}_{\odot}$ so the four galaxies in Figure 6 are not anomalous in mass compared to the rest of the massive disc population. The higher HI mass seen in the disturbed system is consistent with the idea that the gas is likely brought in by a merger and is then used up to form stars, as this merger-driven starburst progresses and the tidal features fade away. The range of HI masses and fractions in massive galaxies from $\mathrm{J} 20$ are shown for comparison and are consistent with that in the disturbed disc.

It is interesting to explore the possible gas fractions of the satellites that trigger the formation of these massive disc galaxies. To do this, we consider the HI content of the disturbed disc ( $10^{10.6} \mathrm{M}_{\odot}$ ). If the hypothesis in $\mathrm{J} 20$ is correct, then this is likely to be more representative of the original gas mass brought in by the minor merger, since, in the relaxed systems, much of the gas is likely to have been used up by the merger-driven star formation episode before we observe the system. Using the median merger mass ratio that creates the massive discs from $\mathrm{J} 20(\sim 1: 4.3)$ implies that the HI fraction of the satellite could be between $\sim 39$ per cent (if one assumes the lower limit of the HI mass and the upper limit of the stellar mass) and $\sim 76$ per cent (if one assumes the upper limit of the HI mass and the lower limit of the stellar mass).

While we caution that these numbers are only indicative they do suggest that the satellites are indeed likely to be gas-rich, consistent with the theoretical predictions. It is worth noting that the satellites themselves have to be relatively massive galaxies. For example, assuming a merger mass ratio of $\sim 1: 4.3$ and a larger galaxy with a stellar mass of $10^{11.4} \mathrm{M}_{\odot}$, the satellite would have a stellar mass of $\left.\sim 10^{10.8} \mathrm{M}_{\odot}\right)$. The lower end of the estimated gas fractions ( 39 per cent) is a factor of $\sim 3$ higher than the median gas fractions for such systems at low redshift (e.g. Zhang et al. 2009; Catinella et al. 

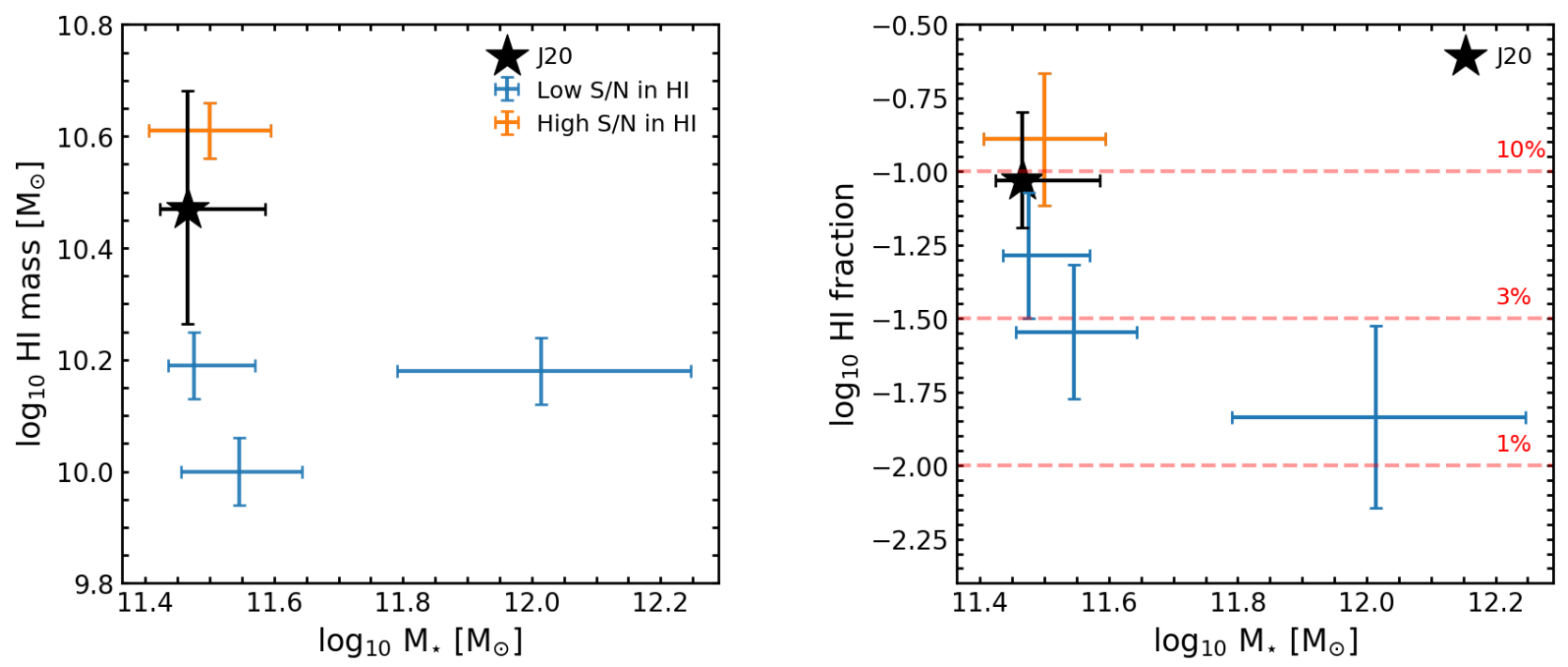

Figure 7. Left: HI mass vs stellar mass for galaxies that have HI detections in the ALFALFA survey. The disturbed disc (lower right-hand panel in Figure 6) has a high signal-to-noise (S/N) detection in ALFALFA (orange) while the other three have low S/N detections (blue). Right: HI fraction (defined as HI mass divided by the sum of the HI and stellar masses) vs stellar mass for the galaxies in the left-hand panel. The median values and distributions for the HI gas masses and gas fractions from the simulated galaxy sample of J20 are shown in black. The HI mass, and the corresponding HI fraction, in the disturbed disc is higher than those in its relaxed counterparts, consistent with the idea that the gas is likely brought in by a merger and is then used up to form stars as this merger-driven starburst progresses and the tidal features fade away, as suggested by J20.

2010; Calette et al. 2018; Hunt et al. 2020). An important caveat in this analysis of HI properties is that it is based on a very small sample of objects. Dedicated deep HI imaging, or future HI surveys which are deeper than those currently available, are required to provide true statistical insights into the empirical gas properties of the massive disc population and consolidate the evidence from the optical images of their minor-merger-driven origin

\section{SUMMARY}

In our standard structure-formation paradigm, the morphological transformation of massive galaxies, from discs to spheroids, is thought to be driven largely by merging. Furthermore, the merger activity experienced by galaxies tends to be a strong function of stellar mass, with the most massive galaxies having the richest merger histories. It is, therefore, surprising that a significant minority ( $>10$ per cent) of massive galaxies at the highest stellar masses $\left(\mathrm{M}_{\star}>10^{11.4} \mathrm{M}_{\odot}\right)$, are in fact discs. In J20, we have used a cosmological hydrodynamical simulation to show that extremely massive discs can form via one of two channels. The dominant channel is a minor merger between a spheroid and a gas-rich satellite, while the secondary channel involves a disc galaxy maintaining its discy morphology due to an anomalously quiet merger history. In this paper, we have studied a large statistical sample of nearby massive galaxies, using data from the SDSS, GALEX, DECaLS and ALFALFA surveys to explore whether the observations support the predicted properties and formation mechanisms of massive discs in J20. Our main conclusions are as follows:

- Massive discs account for $\sim 13$ per cent of massive galaxies in our observational sample, in good agreement with the predicted fraction of discs in $\mathrm{J} 20$ ( $\sim 11$ per cent).

- $\sim 64$ per cent of our massive discs show evidence for tidal features (compared to $\sim 31$ per cent of our massive spheroids). This is a lower limit because deeper images are likely to reveal more galax- ies with tidal features (see Figure 1). In contrast, the tidal feature fraction in low-mass discs, in images that have similar depth and seeing, is $\sim 11-17$ per cent, depending on the specific morphology of the low-mass disc population in question. The presence of tidal features is therefore significantly elevated in massive discs, by at least a factor of 4 compared to their low-mass counterparts, indicating a significant role for merging in the formation of these systems.

- The major-merger fraction for massive galaxies at low redshift is only a few per cent, with tidal features remaining visible for 23 Gyrs at the depth of the DECaLS images. The majority of the interactions seen in our massive discs are, therefore, likely to be minor mergers (i.e. those with mass ratios less than $\sim 1: 4$ ), which are several times more frequent than their major counterparts. This is consistent with the prediction in $\mathbf{J} 20$ that minor mergers are the principal formation mechanism for massive disc galaxies.

- The SFRs of our observed massive discs are in good agreement with those in their simulated counterparts in J20 and also show the predicted elevation compared to that in the massive spheroids. This suggests that the minor mergers in the massive discs are indeed gas-rich, consistent with the hypothesis presented in J20.

- While none of the massive spheroids are detected in HI, four massive discs have $\mathrm{HI}$ detections, of which three are relaxed and one is disturbed. The HI mass (and corresponding HI fraction) in the disturbed disc is higher than those in its relaxed counterparts, consistent with the idea that the gas is brought in by a merger, and is then used up to form stars as this merger-driven starburst progresses and the tidal features fade away.

- Combining the HI content of the disturbed disc $\left(\sim 10^{10.6} \mathrm{M}_{\odot}\right)$, which is likely to be representative of the original gas mass brought in by this particular merger, and the median merger mass ratio predicted in J20 for the massive discs, suggests that the gas fractions of the accreted satellites are likely to be extremely high ( $\sim 40$ per cent or more). Since the satellites themselves are relatively massive galaxies (e.g. assuming that the merger has a mass ratio of $\sim 1: 4$ and the larger galaxy has a stellar mass of $10^{11.4} \mathrm{M}_{\odot}, \mathrm{M}_{\text {sat }}$ is $10^{10.8}$ 
$\mathrm{M}_{\odot}$ ), such gas fractions are a factor of $\sim 3$ higher than the median gas fractions for such systems at low redshift. This is consistent with the predictions of $\mathbf{J} 20$ that massive discs are formed in gas-rich minor mergers.

In summary, the observed properties of nearby massive discs, in terms of their morphological fractions, SFRs and HI properties are in good agreement with the theoretical predictions of J20. This, in turn, suggests that massive disc galaxies in the nearby Universe are likely to have been formed primarily via minor mergers between spheroids and gas-rich satellites.

\section{ACKNOWLEDGEMENTS}

We thank the anonymous referee for many constructive suggestions that improved the quality of the original manuscript. We thank Timothy Davis for many useful discussions. This work was supported in part by the Yonsei University Research Fund (Yonsei Frontier Lab, Young Researcher Supporting Program) of 2021 and by the Korean National Research Foundation (NRF-2020R1A2C3003769). RAJ and SK acknowledge support from the STFC [ST/R504786/1, ST/S00615X/1]. SK acknowledges a Senior Research Fellowship from Worcester College Oxford.

GALEX (Galaxy Evolution Explorer) is a NASA Small Explorer, launched in 2003 April. We gratefully acknowledge NASA's support for construction, operation, and science analysis for the GALEX mission, developed in cooperation with the Centre National d'Etudes Spatiales of France and the Korean Ministry of Science and Technology.

We acknowledge the work of the ALFALFA team in observing, flagging, and processing the ALFALFA data that this work makes use of. The ALFALFA team at Cornell is supported by NSF grants AST-0607007 and AST-1107390 and by the Brinson Foundation.

Funding for the SDSS and SDSS-II has been provided by the Alfred P. Sloan Foundation, the Participating Institutions, the National Science Foundation, the U.S. Department of Energy, the National Aeronautics and Space Administration, the Japanese Monbukagakusho, the Max Planck Society, and the Higher Education Funding Council for England. The SDSS Web Site is http://www.sdss.org/.

The SDSS is managed by the Astrophysical Research Consortium for the Participating Institutions. The Participating Institutions are the American Museum of Natural History, Astrophysical Institute Potsdam, University of Basel, University of Cambridge, Case Western Reserve University, University of Chicago, Drexel University, Fermilab, the Institute for Advanced Study, the Japan Participation Group, Johns Hopkins University, the Joint Institute for Nuclear Astrophysics, the Kavli Institute for Particle Astrophysics and Cosmology, the Korean Scientist Group, the Chinese Academy of Sciences (LAMOST), Los Alamos National Laboratory, the Max-Planck-Institute for Astronomy (MPIA), the MaxPlanck-Institute for Astrophysics (MPA), New Mexico State University, Ohio State University, University of Pittsburgh, University of Portsmouth, Princeton University, the United States Naval Observatory, and the University of Washington.

The Legacy Surveys consist of three individual and complementary projects: the Dark Energy Camera Legacy Survey (DECaLS; Proposal ID \#2014B-0404; PIs: David Schlegel and Arjun Dey), the Beijing-Arizona Sky Survey (BASS; NOAO Prop. ID \#2015A-0801; PIs: Zhou Xu and Xiaohui Fan), and the Mayall z-band Legacy Survey (MzLS; Prop. ID \#2016A-0453; PI: Arjun Dey). DECaLS, BASS and MzLS together include data obtained, respectively, at the Blanco telescope, Cerro Tololo Inter-American Observatory, NSF's NOIRLab; the Bok telescope, Steward Observatory, University of Arizona; and the Mayall telescope, Kitt Peak National Observatory, NOIRLab. The Legacy Surveys project is honored to be permitted to conduct astronomical research on Iolkam Du'ag (Kitt Peak), a mountain with particular significance to the Tohono O'odham Nation.

NOIRLab is operated by the Association of Universities for Research in Astronomy (AURA) under a cooperative agreement with the National Science Foundation.

This project used data obtained with the Dark Energy Camera (DECam), which was constructed by the Dark Energy Survey (DES) collaboration. Funding for the DES Projects has been provided by the U.S. Department of Energy, the U.S. National Science Foundation, the Ministry of Science and Education of Spain, the Science and Technology Facilities Council of the United Kingdom, the Higher Education Funding Council for England, the National Center for Supercomputing Applications at the University of Illinois at Urbana-Champaign, the Kavli Institute of Cosmological Physics at the University of Chicago, Center for Cosmology and AstroParticle Physics at the Ohio State University, the Mitchell Institute for Fundamental Physics and Astronomy at Texas A\&M University, Financiadora de Estudos e Projetos, Fundacao Carlos Chagas Filho de Amparo, Financiadora de Estudos e Projetos, Fundacao Carlos Chagas Filho de Amparo a Pesquisa do Estado do Rio de Janeiro, Conselho Nacional de Desenvolvimento Cientifico e Tecnologico and the Ministerio da Ciencia, Tecnologia e Inovacao, the Deutsche Forschungsgemeinschaft and the Collaborating Institutions in the Dark Energy Survey. The Collaborating Institutions are Argonne National Laboratory, the University of California at Santa Cruz, the University of Cambridge, Centro de Investigaciones Energeticas, Medioambientales y Tecnologicas-Madrid, the University of Chicago, University College London, the DES-Brazil Consortium, the University of Edinburgh, the Eidgenossische Technische Hochschule (ETH) Zurich, Fermi National Accelerator Laboratory, the University of Illinois at Urbana-Champaign, the Institut de Ciencies de l'Espai (IEEC/CSIC), the Institut de Fisica d'Altes Energies, Lawrence Berkeley National Laboratory, the Ludwig Maximilians Universitat Munchen and the associated Excellence Cluster Universe, the University of Michigan, NSF's NOIRLab, the University of Nottingham, the Ohio State University, the University of Pennsylvania, the University of Portsmouth, SLAC National Accelerator Laboratory, Stanford University, the University of Sussex, and Texas A\&M University.

BASS is a key project of the Telescope Access Program (TAP), which has been funded by the National Astronomical Observatories of China, the Chinese Academy of Sciences (the Strategic Priority Research Program "The Emergence of Cosmological Structures" Grant \#XDB09000000), and the Special Fund for Astronomy from the Ministry of Finance. The BASS is also supported by the External Cooperation Program of Chinese Academy of Sciences (Grant \#114A11KYSB20160057), and Chinese National Natural Science Foundation (Grant \#11433005).

The Legacy Survey team makes use of data products from the Near-Earth Object Wide-field Infrared Survey Explorer (NEOWISE), which is a project of the Jet Propulsion Laboratory/California Institute of Technology. NEOWISE is funded by the National Aeronautics and Space Administration.

The Legacy Surveys imaging of the DESI footprint is supported by the Director, Office of Science, Office of High Energy Physics of the U.S. Department of Energy under Contract No. DE-AC0205CH1123, by the National Energy Research Scientific Computing 
Center, a DOE Office of Science User Facility under the same contract; and by the U.S. National Science Foundation, Division of Astronomical Sciences under Contract No. AST-0950945 to NOAO.

\section{DATA AVAILABILITY}

Please contact the authors if the sample of observed massive galaxies used in this study is of interest.

\section{REFERENCES}

Abazajian K. N., et al., 2009, ApJS, 182, 543

Aihara H., et al., 2019, PASJ, p. 106

Barnes J. E., 1992, ApJ, 393, 484

Bernardi M., et al., 2003, AJ, 125, 1817

Blumenthal K. A., et al., 2020, MNRAS, 492, 2075

Bournaud F., Jog C. J., Combes F., 2007, A\&A, 476, 1179

Brinchmann J., Charlot S., White S. D. M., Tremonti C., Kauffmann G., Heckman T., Brinkmann J., 2004, MNRAS, 351, 1151

Buitrago F., Conselice C. J., Epinat B., Bedregal A. G., Grützbauch R., Weiner B. J., 2014, MNRAS, 439, 1494

Calette A. R., Avila-Reese V., Rodríguez-Puebla A., Hernández-Toledo H., Papastergis E., 2018, Rev. Mex. Astron. Astrofis., 54, 443

Catinella B., et al., 2010, MNRAS, 403, 683

Cattaneo A., Mamon G. A., Warnick K., Knebe A., 2011, A\&A, 533, A5

Conselice C. J., 2006, ApJ, 638, 686

Conselice C. J., Bluck A. F. L., Mortlock A., Palamara D., Benson A. J., 2014, MNRAS, 444, 1125

Darg D. W., et al., 2010, MNRAS, 401, 1043

Dey A., et al., 2019, AJ, 157, 168

Di Matteo P., Combes F., Melchior A.-L., Semelin B., 2007, A\&A, 468, 61

Dubois Y., Gavazzi R., Peirani S., Silk J., 2013, MNRAS, 433, 3297

Dubois Y., et al., 2014, MNRAS, 444, 1453

Dubois Y., Peirani S., Pichon C., Devriendt J., Gavazzi R., Welker C., Volonteri M., 2016, MNRAS, 463, 3948

Faber S. M., et al., 2007, ApJ, 665, 265

Font A. S., McCarthy I. G., Le Brun A. M. C., Crain R. A., Kelvin L. S., 2017, preprint, (arXiv:1710.00415)

Governato F., et al., 2009, MNRAS, 398, 312

Haynes M. P., et al., 2011, AJ, 142, 170

Hilz M., Naab T., Ostriker J. P., 2013, MNRAS, 429, 2924

Hood C. E., Kannappan S. J., Stark D. V., Dell'Antonio I. P., Moffett A. J., Eckert K. D., Norris M. A., Hendel D., 2018, ApJ, 857, 144

Hopkins P. F., Cox T. J., Younger J. D., Hernquist L., 2009, ApJ, 691, 1168

Hunt L. K., Tortora C., Ginolfi M., Schneider R., 2020, A\&A, 643, A180

Jackson R. A., Martin G., Kaviraj S., Laigle C., Devriendt J. E. G., Dubois Y., Pichon C., 2020, MNRAS, 494, 5568

Jackson R. A., et al., 2021, MNRAS, 502, 1785

Jogee S., et al., 2009, ApJ, 697, 1971

Kaviraj S., 2010, MNRAS, 406, 382

Kaviraj S., 2014a, MNRAS, 437, L41

Kaviraj S., 2014b, MNRAS, 440, 2944

Kaviraj S., Tan K.-M., Ellis R. S., Silk J., 2011, MNRAS, 411, 2148

Kaviraj S., Devriendt J., Dubois Y., Slyz A., Welker C., Pichon C., Peirani S., Le Borgne D., 2015, MNRAS, 452, 2845

Kaviraj S., et al., 2017, MNRAS, 467, 4739

Kaviraj S., Martin G., Silk J., 2019, MNRAS, 489, L12

Li C., White S. D. M., 2009, MNRAS, 398, 2177

Lofthouse E. K., Kaviraj S., Conselice C. J., Mortlock A., Hartley W., 2017, MNRAS, 465, 2895

López-Sanjuan C., Balcells M., Pérez-González P. G., Barro G., GarcíaDabó C. E., Gallego J., Zamorano J., 2010, ApJ, 710, 1170

Lotz J. M., Jonsson P., Cox T. J., Croton D., Primack J. R., Somerville R. S., Stewart K., 2011, ApJ, 742, 103
Mancillas B., Duc P.-A., Combes F., Bournaud F., Emsellem E., Martig M., Michel-Dansac L., 2019, A\&A, 632, A122

Martig M., et al., 2021, MNRAS, 508, 2458

Martin G., Kaviraj S., Devriendt J. E. G., Dubois Y., Pichon C., 2018, MNRAS, 480, 2266

Martin G., et al., 2019, MNRAS, 485, 796

Martin G., et al., 2021, MNRAS, 500, 4937

McIntosh D. H., Guo Y., Hertzberg J., Katz N., Mo H. J., van den Bosch F. C., Yang X., 2008, MNRAS, 388, 1537

Monachesi A., Bell E. F., Radburn-Smith D. J., Bailin J., de Jong R. S., Holwerda B., Streich D., Silverstein G., 2016, MNRAS, 457, 1419

Morrissey P., et al., 2007, ApJS, 173, 682

Mundy C. J., Conselice C. J., Duncan K. J., Almaini O., Häußler B., Hartley W. G., 2017, MNRAS, 470, 3507

Nelson D., et al., 2019, Computational Astrophysics and Cosmology, 6, 2

Ogle P. M., Lanz L., Nader C., Helou G., 2016, The Astrophysical Journal, 817,109

Ogle P. M., Lanz L., Appleton P. N., Helou G., Mazzarella J., 2019, ApJS, 243,14

Oser L., Ostriker J. P., Naab T., Johansson P. H., Burkert A., 2010, ApJ, 725, 2312

Peschken N., Łokas E. L., Athanassoula E., 2019, arXiv e-prints, p. arXiv: 1909.01033

Robertson B., Bullock J. S., Cox T. J., Di Matteo T., Hernquist L., Springel V., Yoshida N., 2006, ApJ, 645, 986

Rodriguez-Gomez V., et al., 2015, MNRAS, 449, 49

Ryan R. E. J., et al., 2012, ApJ, 749, 53

Salim S., et al., 2016, ApJS, 227, 2

Shibuya T., Ouchi M., Harikane Y., 2015, ApJS, 219, 15

Springel V., Hernquist L., 2005, ApJ, 622, L9

Toomre A., 1977, in Tinsley B. M., Larson D. Campbell R. B. G., eds, Evolution of Galaxies and Stellar Populations. p. 401

Welker C., Dubois Y., Pichon C., Devriendt J., Chisari N. E., 2018, A\&A, 613, A4

Wright A. H., et al., 2017, MNRAS, 470, 283

Wuyts S., et al., 2011, ApJ, 742, 96

Yang X., Mo H. J., van den Bosch F. C., Pasquali A., Li C., Barden M., 2007, ApJ, 671, 153

York D. G., et al., 2000, AJ, 120, 1579

Zeng G., Wang L., Gao L., 2021, MNRAS, 507, 3301

Zhang W., Li C., Kauffmann G., Zou H., Catinella B., Shen S., Guo Q., Chang R., 2009, MNRAS, 397, 1243

This paper has been typeset from a $\mathrm{T}_{\mathrm{E}} \mathrm{X} / \mathrm{LAT}_{\mathrm{E}} \mathrm{X}$ file prepared by the author. 